\title{
Drug Release Kinetics from Polymer Matrix through Fractal Approximation of Motion
}

\author{
S. Băcăiţă, ${ }^{1,2}$ C. Urîtu, ${ }^{3}$ M. Popa, ${ }^{1}$ A. Uliniuc, ${ }^{1}$ C. Peptu, ${ }^{1}$ and M. Agop ${ }^{2,4}$ \\ ${ }^{1}$ Department of Natural and Synthetic Polymers, Faculty of Chemical Engineering and Environmental Protection, \\ "Gheorghe Asachi” Technical University, Prof. Dr. Docent Dimitrie Mangeron Road, No. 73, 700050 Iasi, Romania \\ ${ }^{2}$ Physics Department, Faculty of Machine Manufacturing and Industrial Management, "Gheorghe Asachi" Technical University, \\ Prof. Dr. Docent Dimitrie Mangeron Road, No. 59A, 700050 Iasi, Romania \\ ${ }^{3}$ Centre of Advanced Researches for Bionanoconjugates and Biopolymers, "Petru Poni" Institute of Macromolecular Chemistry, \\ Grigore Ghica Voda Avenue, No. 41A, 700487 Iasi, Romania \\ ${ }^{4}$ Lasers, Atoms and Molecules Physics Laboratory, University of Science and Technology, Villeneuve d'Ascq, 59655 Lille, France
}

Correspondence should be addressed to M. Agop, m.agop@yahoo.com

Received 10 February 2012; Revised 4 March 2012; Accepted 5 March 2012

Academic Editor: Metin Ak

Copyright (C) 2012 S. Băcăiţă et al. This is an open access article distributed under the Creative Commons Attribution License, which permits unrestricted use, distribution, and reproduction in any medium, provided the original work is properly cited.

The present paper analyzes the process of drug release from polymer matrix. This process has been considered as fractal polymer process. Since complexity of physical processes is replaced by fractality, the paper studies the process through fractal approach. In drug dynamics, fractal "diffusion" equation can be obtained through fractal approximation of motion. All experimental release curves have been best demonstrated by Weibull relation (which was, in its turn, also demonstrated). Weibull parameters are related to the fractal dimension of drug release kinetics from a polymer matrix. Such a dimension can characterize and measure the complexity of the system. In the above-mentioned context, some experimental results of our researchers are presented and analyzed by comparing them with Peppas relation, a basic law in the description of drug release kinetics. Consequently, experimental data for Weibull relation are better correlated with certain resulting factors. At the same time, some conclusions regarding the phenomena involved in the process are considered as being based on the approach.

\section{Introduction}

The adequate description of real, natural, and artificial objects is restricted by the mere use of Euclidean geometry, that is, the description of integer-dimensioned objects. In such perspective, this happens because numerous objects with noninteger dimensions, such as plants, galaxies, population patterns, and crystal growth, are left beyond analytical purpose. The characteristics of such objects can be described by means of fractal geometry $[1,2]$. Natural and synthetic polymers considered as fractal objects are also included in the above category. Their main structural unit, the macromolecular coil, is known to be a fractal with typical fractal behavior [3-8].

The main idea of the paper focuses on this type of processes in which polymer fractality is responsible for drug release from various polymer matrixes. In what follows, we shall produce arguments to sustain the above statement.
The experimental drug release kinetics indicate that drug loaded polymer matrix structures are thermodynamically unstable and evolve towards equilibrium. Specific parameters for each structure (drug type, incorporated drug dose(s), types and amounts of excipients, preparation technique, environmental conditions during drug release, and geometry and dimensions of drug delivery system) will lead to different evolutive trajectories, considered as consequences of internal collective processes. Thermodynamically nonbalanced processes generate the formation of fractal structures $[5,9]$. That is why fractal structures appear in drug release environment, too.

Drug release phenomena (water penetration into the device, drug and excipient dissolution, phase transitions, drug and/or polymer degradation, polymer swelling, physical drug-excipient interactions, and chemical reactions of drug, excipients, and/or water) are complex processes. Nevertheless, mathematical expressions pharmaceutics uses 
for the description of drug release kinetics are rather simple, namely Higuchi, Ritger-Peppas, Peppas-Sahlin, and Alfrey power laws [10]. The structure whose dynamics is ruled by power laws tends to critically organize itself [11], as fractals do.

Thus, the question of whether or not fractal analysis should be used in the description of the structure and evolution of such a structure, that is, drug-loaded polymer matrix, is not a matter of scientific choice, but a consequence of a correct approach.

This is the reason why we will analyze this process considering that fractality can replace the complexity of physical processes. Moreover, it will be no longer necessary to use the entire classical "arsenal" of quantities of standard physics (differentiable physics). Physical systems will behave as special "fluids" that lack interaction. Thereby, we introduced fractal approximation of motion in the study of the complex dynamics of physical systems, taking into account that drug trajectories are continuous, but non-differentiable curves, called fractal curves. Interactions are dealt with in the above-mentioned manner by a physical model, which is the scale relativity theory (SRT) $[12,13]$. Accordingly, we will obtain a fractal "diffusion" equation and deduce Weibull law that, in comparison with power laws, better describes all experimental release curves [14], thus eliminating all possible critical opinions regarding the absence of kinetics in the use of Weibull's law or the nonphysical nature of its parameters [15].

This paper is structured as follows: Section 2 presents general notions on the fractal approximation of motion through scale relativity theory. Fractal "diffusion" equations, both for Fick type "diffusion" and for anomalous type "diffusion," as well as Weibull relation are exhibited in Section 3. Experimental results of our theoretical model are presented in Section 4. Section 5 comprises the conclusions.

\section{Fractal Approximation of Motion Applied in Drug Release Process}

Drug-loaded polymer matrix can be included in the category of complex dynamic systems which display nonlinear behavior, self-similarity, and self-structure. At the same time, these systems intensely fluctuate at all possible scales [9-21]. Since fractality appears to be the universal feature of such systems, it is essential to construct fractal physics $[13,18]$ when describing such evolution.

2.1. Non-differentiability Consequences in Drug Release Process. Drug release process is a very complex phenomenon, with many significant variables in the evolution of the system. That is why we assume that this type of process takes place on continuous, but non-differentiable curves (fractal curves). Non-differentiability implies the following $[13,18]$.

(i) A continuous and non-differentiable curve (or almost nowhere differentiable) is explicitly scale dependent, and its length tends to infinity, when the scale interval tends to zero. In other words, a continuous and non-differentiable space is a fractal one, in that general meaning Mandelbrot gave to the concept [11].

(ii) There is an infinity of fractal curves (geodesics) that connects its points (or starting from any point) and this is applicable for all scales.

(iii) Local differential time reflection invariance breaking: the time derivative of relative variation for the released drug $Q$ can be written twice:

$$
\begin{aligned}
\frac{d Q}{d t} & =\lim _{\Delta t \rightarrow 0_{+}} \frac{Q(t+\Delta t)-Q(t)}{\Delta t}, \\
\frac{d Q}{d t} & =\lim _{\Delta t \rightarrow 0_{-}} \frac{Q(t)-Q(t-\Delta t)}{\Delta t} .
\end{aligned}
$$

Both definitions are equivalent for the differentiable case. As regards non-differentiable situations, these definitions are no longer valid, since limits cannot anymore be defined. Within fractal theory, physics is related to the behavior of the function during the "zoom" operation on the time resolution $\delta t$, here identified with the differential element $d t$ (substitution principle), which is considered as an independent variable. The standard concentration field $Q(t)$ is therefore replaced by fractal concentration field $Q(t, d t)$, explicitly dependent on the time resolution interval. The characteristic derivative of this interval is undefined only at that unnoticed limit $d t \rightarrow 0$ which is described by a fractal function. Consequently, this will lead to the definition of the two derivatives of the fractal concentration field $Q$ as explicit functions of $t$ and $d t$ variables:

$$
\begin{aligned}
& \frac{d_{+} Q}{d t}=\lim _{\Delta t \rightarrow 0_{+}} \frac{Q(t+\Delta t, \Delta t)-Q(t, \Delta t)}{\Delta t}, \\
& \frac{d_{-} Q}{d t}=\lim _{\Delta t \rightarrow 0_{-}} \frac{Q(t, \Delta t)-Q(t-\Delta t, \Delta t)}{\Delta t} .
\end{aligned}
$$

"+" corresponds to the forward process and "-" to the backward one.

(iv) The differential of the coordinates, $d_{ \pm} X(t, d t)$, can be decomposed as follows:

$$
d_{ \pm} X(t, d t)=d_{ \pm} x(t)+d_{ \pm} \xi(t, d t)
$$

where $d_{ \pm} x(t)$ is the "classical part" and $d_{ \pm} \xi(t, d t)$ is the "fractal part," depending on resolution. The differential of the "fractal part" components $\xi^{i}(t, d t), i=\overline{1,3}$, satisfies the relation (the fractal equation) [22-24]

$$
d_{ \pm} \xi^{i}=\lambda_{ \pm}^{i}(d t)^{1 / D_{F}}
$$

where $\lambda_{ \pm}^{i}$ are constant coefficients, and $D_{F}$ is the fractal dimension. For fractal dimension, we can use any definition (Kolmogorov, Hausdorff [1, 13, 25-27], etc.), but once a certain definition is admitted, it should be kept up to the end of analysis. 
(v) The local differential time reflection invariance is recovered by combining the two derivatives, $d_{+} / d t$ and $d_{-} / d t$, in the complex operator

$$
\frac{\hat{\partial}}{\partial t}=\frac{1}{2}\left(\frac{d_{+}+d_{-}}{d t}\right)-\frac{i}{2}\left(\frac{d_{+}-d_{-}}{d t}\right) .
$$

When this operator is applied to the "position vector," a complex speed yields

$$
\hat{\mathbf{V}}=\frac{\hat{d} \mathbf{X}}{d t}=\mathbf{V}-i \mathbf{U}
$$

with

$$
\mathbf{V}=\frac{\mathbf{V}_{+}+\mathbf{V}_{-}}{2}, \quad \mathbf{U}=\frac{\mathbf{V}_{+}-\mathbf{V}_{-}}{2}
$$

The real part $\mathbf{V}$ of the complex speed $\hat{\mathbf{V}}$ represents the standard classical speed, which is independent of resolution, while the imaginary part $\mathbf{U}$ is a new quantity arising from fractality, which is resolution dependent.

(vi) The average values of the quantities must be considered in the sense of a generalized statistical fluid. Particularly, the average of $d_{ \pm} \mathbf{X}$ is

$$
\left\langle d_{ \pm} \mathbf{X}\right\rangle=d_{ \pm} \mathbf{X}
$$

with

$$
\left\langle d_{ \pm} \xi\right\rangle=0
$$

2.2. Covariant Total Derivative in Drug Release Process. Let us now admit that the curve (continuous, but nondifferentiable), describing the drug release, is immersed in a three-dimensional space, and that $\mathbf{X}$ of components $X^{i}(i=\overline{1,3})$ is the position vector of a point on the curve. Let us also consider the fractal concentration field $Q(X, t)$ and expand its total differential up to second order

$$
d_{ \pm} Q=\frac{\partial Q}{\partial t} d t+\nabla Q \cdot d_{ \pm} \mathbf{X}+\frac{1}{2} \frac{\partial^{2} Q}{\partial X^{i} \partial X^{j}} d_{ \pm} X^{i} d_{ \pm} X^{j}
$$

The relations (10) are manifold valid in any point of the space and, implicitly, for the points $\mathbf{X}$ on the fractal curve which we have selected in relations (10). Hence, the forward and backward average values of this relation take the form

$$
\begin{aligned}
\left\langle d_{ \pm} Q\right\rangle= & \left\langle\frac{\partial Q}{\partial t} d t\right\rangle+\left\langle\nabla Q \cdot d_{ \pm} \mathbf{X}\right\rangle \\
& +\frac{1}{2}\left\langle\frac{\partial^{2} Q}{\partial X^{i} \partial X^{j}} d_{ \pm} X^{i} d_{ \pm} X^{j}\right\rangle .
\end{aligned}
$$

We formulate the following stipulation [22-24]: the mean value of the fractal concentration field $Q$ and its derivatives coincide with themselves and the differentials $d_{ \pm} X^{i}$ and $d t$ are independent. Therefore, the average of their products coincides with the product of the averages. Thus, using (3) with the property (9), (11) becomes

$$
\begin{aligned}
d_{ \pm} Q= & \frac{\partial Q}{\partial t} d t+\nabla Q \cdot d_{ \pm} \mathbf{x} \\
& +\frac{1}{2} \frac{\partial^{2} Q}{\partial X^{i} \partial X^{j}}\left(d_{ \pm} x^{i} d_{ \pm} x^{j}+\left\langle d_{ \pm} \xi^{i} d_{ \pm} \xi^{j}\right\rangle\right) .
\end{aligned}
$$

Even the average value of the fractal coordinate $d_{ \pm} \xi^{i}$ is null (see (9)), whilst for the higher order of the fractal coordinate average, the situation can be different. Firstly, let us focus on the mean $\left\langle d_{ \pm} \xi^{i} d_{ \pm} \xi^{j}\right\rangle$. If $i \neq j$, this average is zero due the independence of $d_{ \pm} \xi^{i}$ and $d_{ \pm} \xi^{j}$. So, using (4), we can write

$$
\left\langle d_{ \pm} \xi^{i} d_{ \pm} \xi^{j}\right\rangle=\lambda_{ \pm}^{i} \lambda_{ \pm}^{j}(d t)^{\left(2 / D_{F}\right)-1} d t
$$

Then, if we divide by $d t$ and neglect the terms which contain differential factors (for details on the method, see $[16,17]),(12)$ may be written under the form

$$
\frac{d_{ \pm} Q}{d t}=\frac{\partial Q}{\partial t}+\mathbf{V}_{ \pm} \cdot \nabla Q+\frac{1}{2} \frac{\partial^{2} Q}{\partial X^{i} \partial X^{j}} \lambda_{ \pm}^{i} \lambda_{ \pm}^{j}(d t)^{\left(2 / D_{F}\right)-1}
$$

These relations also allow us to define the operator

$$
\frac{d_{ \pm}}{d t}=\frac{\partial}{\partial t}+\mathbf{V}_{ \pm} \cdot \nabla+\frac{1}{2} \frac{\partial^{2}}{\partial X^{i} \partial X^{j}} \lambda_{ \pm}^{i} \lambda_{ \pm}^{j}(d t)^{\left(2 / D_{F}\right)-1}
$$

Under these circumstances, let us calculate $(\hat{\partial} Q / \partial t)$. Taking into account (15), (5), and (6), we obtain

$$
\begin{aligned}
\frac{\partial \hat{Q}}{\partial t}= & \frac{\partial Q}{\partial t}+\hat{\mathbf{V}} \cdot \nabla Q+\frac{(d t)^{\left(2 / D_{F}\right)-1}}{4} \\
& \times\left[\left(\lambda_{+}^{i} \lambda_{+}^{j}+\lambda_{-}^{i} \lambda_{-}^{j}\right)-i\left(\lambda_{+}^{i} \lambda_{+}^{j}-\lambda_{-}^{i} \lambda_{-}^{j}\right)\right] \frac{\partial^{2} Q}{\partial X^{i} \partial X^{j}}
\end{aligned}
$$

This relation also allows the definition of the fractal operator

$$
\begin{aligned}
\frac{\hat{\partial}}{\partial t}= & \frac{\partial}{\partial t}+\hat{\mathbf{V}} \cdot \nabla+\frac{(d t)^{\left(2 / D_{F}\right)-1}}{4} \\
& \times\left[\left(\lambda_{+}^{i} \lambda_{+}^{j}+\lambda_{-}^{i} \lambda_{-}^{j}\right)-i\left(\lambda_{+}^{i} \lambda_{+}^{j}-\lambda_{-}^{i} \lambda_{-}^{j}\right)\right] \frac{\partial^{2}}{\partial X^{i} \partial X^{j}}
\end{aligned}
$$

Particularly, by choosing

$$
\lambda_{+}^{i} \lambda_{+}^{j}=-\lambda_{-}^{i} \lambda_{-}^{j}=2 \mathfrak{D} \delta^{\mathrm{ij}},
$$

we obtain the following results:

(i) the relation (13) becomes

$$
\left\langle d_{ \pm} \xi^{i} d_{ \pm} \xi^{j}\right\rangle=2 \mathfrak{D}(d t)^{\left(2 / D_{F}\right)-1} d t
$$


(ii) the fractal operator (17) takes the form [22-24]

$$
\frac{\hat{\partial}}{\partial t}=\frac{\partial}{\partial t}+\hat{\mathbf{V}} \cdot \nabla-i \mathfrak{D}(d t)^{\left(2 / D_{F}\right)-1} \Delta
$$

(iii) Nottale's model is formulated in the fractal dimension $D_{F}=2$, that is, for movements on Peano curves and for Wiener's stochastic processes [1, 13, 25-27].

\section{Generalized Principle of Scale Covariance: Fractal "Diffusion" Type Equation}

We now apply the principle of scale covariance and postulate that the transition from classical (differentiable) to "fractal" physics can be implemented by means of replacing the standard time derivative operator $d / d t$ with the complex one $\hat{\partial} / \partial t$ (this result is a generalization of Notalle's scale covariance principle as in [13]). As a consequence, we can now write the fractal "diffusion" type equation in its covariant form

$$
\frac{\hat{\partial} Q}{d t}=\frac{\partial Q}{\partial t}+(\hat{\mathbf{V}} \cdot \nabla) Q-i \mathfrak{D}(d t)^{\left(2 / D_{F}\right)-1} \Delta Q=0 .
$$

This means that at any point of a fractal curve, the local temporal term $\partial_{t} Q$, the nonlinearly "convective" term $(\hat{\mathbf{V}} \cdot \nabla) Q$, and the dissipative one $\Delta Q$ equilibrate.

3.1. Standard "Diffusion" Type Equation. Separating the real part from the imaginary one in (21), that is,

$$
\begin{gathered}
\frac{\partial Q}{\partial t}+\mathbf{V} \cdot \nabla Q=0, \\
-\mathbf{U} \cdot \nabla Q=\mathfrak{D}(d t)^{\left(2 / D_{F}\right)-1} \Delta Q,
\end{gathered}
$$

we can add these two equations and obtain a generalized "diffusion" type law in the form

$$
\frac{\partial Q}{\partial t}+(\mathbf{V}-\mathbf{U}) \cdot \nabla Q=\mathfrak{D}(d t)^{\left(2 / D_{F}\right)-1} \Delta Q
$$

The standard "diffusion" law, that is,

$$
\frac{\partial Q}{\partial t}=D \Delta Q
$$

results from (23) the following assertions:

(i) diffusion paths are Peano type fractal curves. In other words, the fractal dimension of the fractal curves is $D_{F}=2$. Moreover, the average values (19) are defined through Wiener's stochastic processes [1, 13, 25-27], that is,

$$
\left\langle d_{ \pm} \xi^{i} d_{ \pm} \xi^{j}\right\rangle=2 \mathfrak{D} d t
$$

(ii) the movements at differentiable and non-differentiable scales are synchronous, that is, $\mathbf{V}=\mathbf{U}$, (iii) the structure coefficient $\mathfrak{D}$, that characterizes fractalnonfractal transition, identifies with diffusion coefficient, that is,

$$
\mathfrak{D} \equiv \mathbf{D}
$$

This kind of "diffusion" is analyzed in [28, 29], a situation for which Papadopoulou et al. [30] calculated the value of the constant $b$ and obtained $b \cong 1$, equivalent with $D_{F}=2$.

3.2. Anomalous "Diffusion" Type Equation: Weibull Relation. The anomalous diffusion law results from (23) on the following assumptions:

(i) diffusion paths are fractal curves with fractal dimension $D_{F} \neq 2$,

(ii) time resolution $\delta t$ is identified with the differential element $d t$, that is, in this case, the substitution principle can be also applied,

(iii) movements at differentiable and non-differentiable scales are synchronous, that is, $\mathbf{V}=\mathbf{U}$.

Then, (23) can be written as follows:

$$
\frac{\partial Q}{\partial t}=\mathfrak{D}(d t)^{\left(2 / D_{F}\right)-1} \Delta Q .
$$

For the one-dimensional case, applying the variable separation method [31]

$$
Q(t, \mathbf{x})=T(t) \cdot X(\mathbf{x}),
$$

with standard initial and boundary conditions

$$
\begin{array}{r}
Q(t, 0)=0, \quad Q(t, L)=0, \quad Q(0, x)=F(x) \\
0 \leq x \leq L
\end{array}
$$

implies

$$
\begin{aligned}
\frac{1}{\mathfrak{D}(d t)^{\left(2 / D_{F}\right)-1}} \frac{1}{T(t)} \frac{d T(t)}{d t} & =\frac{1}{X(x)} \frac{d^{2} X(x)}{d x^{2}} \\
& =-m^{2}=-\left(\frac{n \pi}{L}\right)^{2}, \quad n=1,2,
\end{aligned}
$$

where $L$ is a system characteristic length, and $m$ is a separation constant, dependent on diffusion order $n$.

Accepting the viability of the substitution principle (see Section 2.1), from (30), through integration, it follows that

$$
\ln T=-m^{2} \mathfrak{D} \int(d t)^{\left(2 / D_{F}\right)} .
$$

According to certain results of the fractional integraldifferential calculations [32, 33], (31) becomes

$\ln T=-\frac{m^{2} \mathfrak{D}}{\Gamma\left(\left(2 / D_{F}\right)+1\right)} t^{2 / D_{F}}, \quad \Gamma\left(\frac{2}{D_{F}}\right)=\int_{0}^{\infty} x^{\left(2 / D_{F}\right)-1} e^{-x} d x$. 
Moreover, (32) can be written under the form

$$
T(t)=\exp \left[-\frac{m^{2} \mathfrak{D}}{\Gamma\left(\left(2 / D_{F}\right)+1\right)} t^{2 / D_{F}}\right] .
$$

The time-dependent relative variation of concentrations is defined as

$$
T(t)=\frac{Q_{\infty}-Q_{t}}{Q_{\infty}},
$$

where $Q_{t}$ and $Q_{\infty}$ are cumulative amounts of drug released at time $t$ and infinite time.

From (33) and (34), it follows that

$$
\frac{Q_{t}}{Q_{\infty}}=1-\exp \left[-\frac{m^{2} \mathfrak{D}}{\Gamma\left(\left(2 / D_{F}\right)+1\right)} t^{2 / D_{F}}\right],
$$

equation similar to Weibull relation $Q_{t} / Q_{\infty}=1-\exp \left(-a t^{b}\right)$, where $a$ and $b$ represent characteristic constants for each system that can be defined by

$$
\begin{gathered}
a=\frac{m^{2} \mathfrak{D}}{\Gamma\left(\left(2 / D_{F}\right)+1\right)}=\left(\frac{n \pi}{L}\right)^{2} \frac{\mathfrak{D}}{\Gamma\left(\left(2 / D_{F}\right)+1\right)}, \\
b=\frac{2}{D_{F}} .
\end{gathered}
$$

We can notice that both $a$ and $b$ constants are functions of fractal dimension of the curves on which drug release mechanism takes place. This represents the complexity and nonlinearity dynamics of the system. Moreover, constant $a$ is also dependent on the "diffusion" order $n$.

Let us note that the fractal processes $[1,13,25-27]$ given by (21) with $D_{F} \neq 2$ are considered as being "anomalous diffusion" (subdiffusion for $D_{F}<2$ and superdiffusion for $D_{F}>2$ ). The "Fokker-Planck equations" for anomalous diffusion do not usually have the form of an ordinary diffusion equation. Indeed, it is well known that the "FokkerPlanck equations" for anomalous diffusion take the form of fractional derivative equations, called fractional FokkerPlanck equations [25-27].

This new approach will be presently applied in the analysis of one polymer-drug type system that has been obtained by our researchers.

\section{Experimental Results and Discussion}

This paragraph presents some experimental results obtained by our researchers, for different kind of polymer matrices.

4.1. Chloramphenicol-Loaded GEL-PVA Microparticles. Gelatin and poly(vinyl alcohol) (GEL-PVA) microparticles crosslinked with glutaraldehyde (GA), for samples with different amount of cross-linking agent $(2 \%, 6 \%, 8 \%, 10 \%, \ldots$, the sample code indicates the cross-linking amount: for example, GA2 represents a sample with $2 \%$ cross linking amount)

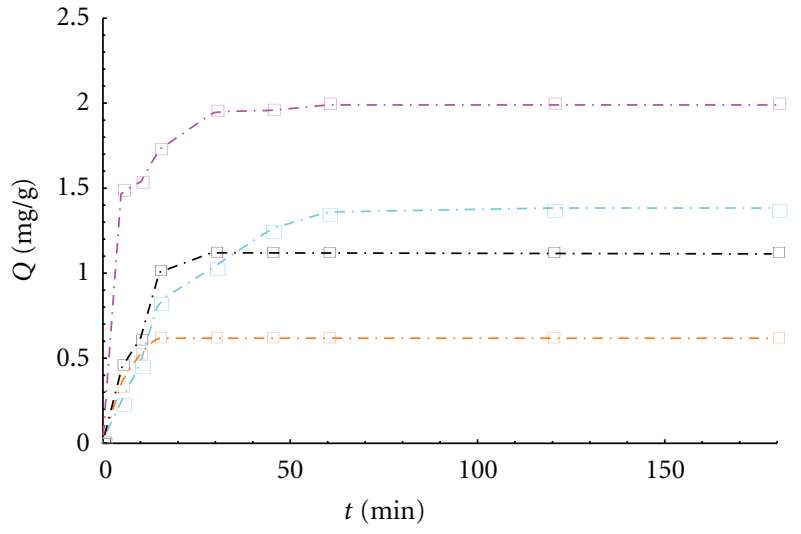

$\begin{array}{llll}- & \text { Experimental points GA6 } \\ - & - & \text { Experimental points GA8 } \\ - & \text { Experimental points GA2 } & - & \text { Experimental points GA10 }\end{array}$

FIgure 1: Chloramphenicol release kinetics from GEL-PVA microparticles.

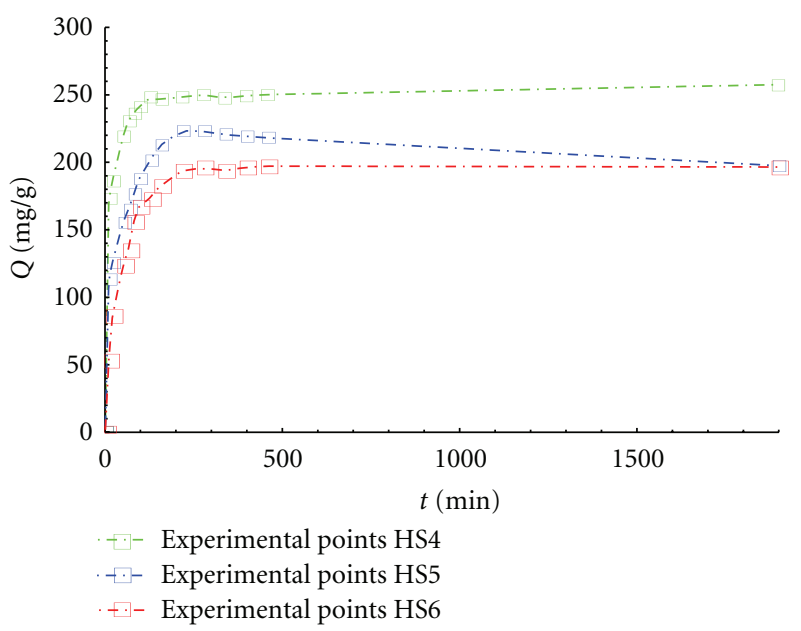

FIGURE 2: Levofloxacin release kinetics from starch-based hydrogels.

loaded with chloramphenicol were tested (details regarding materials and experimental protocol can be found in [34]).

The experimental points of release kinetics are shown in Figure 1.

4.2. Levofloxacin-Loaded Starch-Based Hydrogels. Hydrogels with controlled hydrophilicity were obtained from new amphiphilic starch-polyester graft copolymer and unmodified starch with fatty acid chains, cross-linked with citric acid, at different cross-linking time $(4 \mathrm{~h}, 5 \mathrm{~h}, 6 \mathrm{~h}, \ldots$, the sample code indicates the cross-linking time: for example, HS4 represents a sample with $4 \mathrm{~h}$ cross-linking time). The samples were loaded with levofloxacin, and their release kinetics was studied (details regarding materials and experimental protocol can be found in [35]).

The experimental points of release kinetics are shown in Figure 2. 


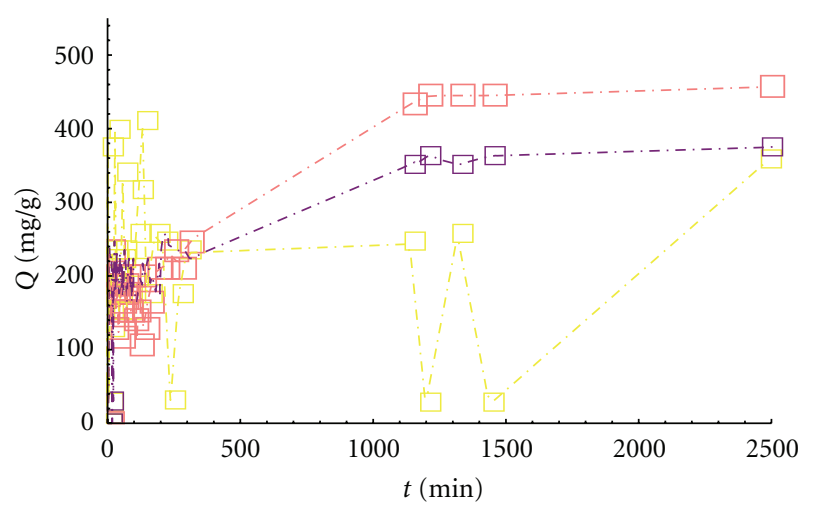

Experimental points GC $10-90$

- - Experimental points GC 30-70

- - Experimental points GC 50-50

Figure 3: Adrenaline release kinetics from GEL-CHI microparticles.

4.3. Adrenaline-Loaded GEL-CHI Microparticles. Gelatin and chitosan (GEL-CHI) microparticles were prepared within two-step cross-linking process in an emulsion-phase separation system cross-linked with glutaraldehyde (GA). Samples were prepared with different gelatin: chitosan-gelatin amount ratios of $(10 \%-90 \%, 30 \%-70 \%, 50 \%-50 \%, \ldots$, the sample code indicates the gelatin/chitosan ratio: for example, GC 10-90 represents a $10 \%$ gelatin and $90 \%$ chitosan sample).

The samples were loaded with adrenaline, and their release kinetics was studied (details regarding materials and experimental protocol can be found in [36]).

The experimental points of release kinetics are shown in Figure 3.

4.4. Experimental Results Analysis and Discussions. As a result of different experimental protocols, all the above drug carriers represent differentiated polymer matrices. Despite this, they have qualitatively similar time behavior. Therefore, qualitative analysis should be performed according to the loaded drug amount that is directly proportional to the released drug amount.

These results have been analyzed matching experimental data with Peppas and Weibull laws, as it has been demonstrated in the previous paragraph. As a result, for each of the samples (see Table 1), we obtained Peppas and Weibull parameters, the correlation factors, and release kinetics fractal dimension.

The first observation refers to the correlation coefficient between the experimental curves and Weibull fitted curve, which proved to be a very good one, even better than in the case of Peppas curve. This means that the entire release process can be better described by Weibull law as compared to Peppas law, thus showing the wide applicability of the former (the best values for correlation factors are italicized, in Table 1). Experimental Weibull curves for HS and GA samples are graphically illustrated in Figure 4.

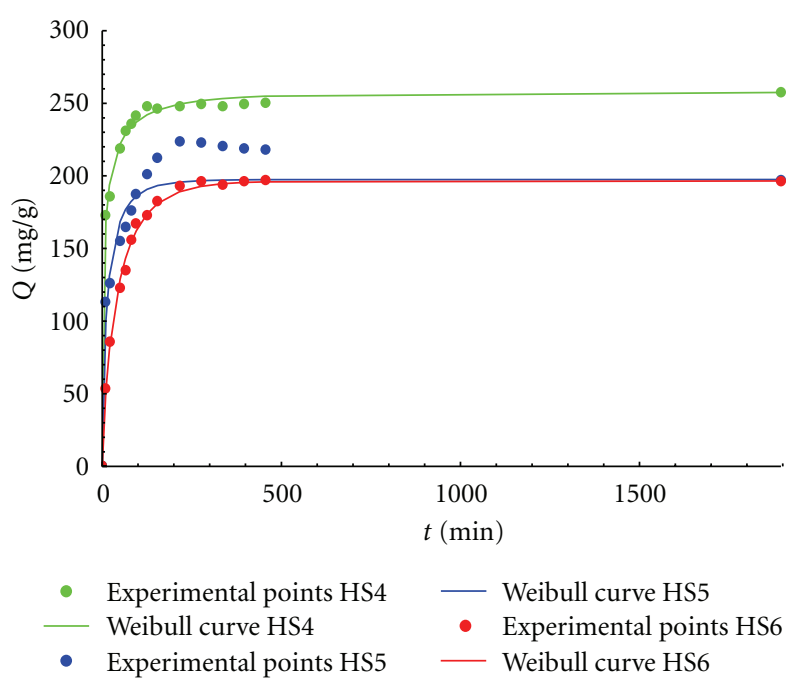

(a)

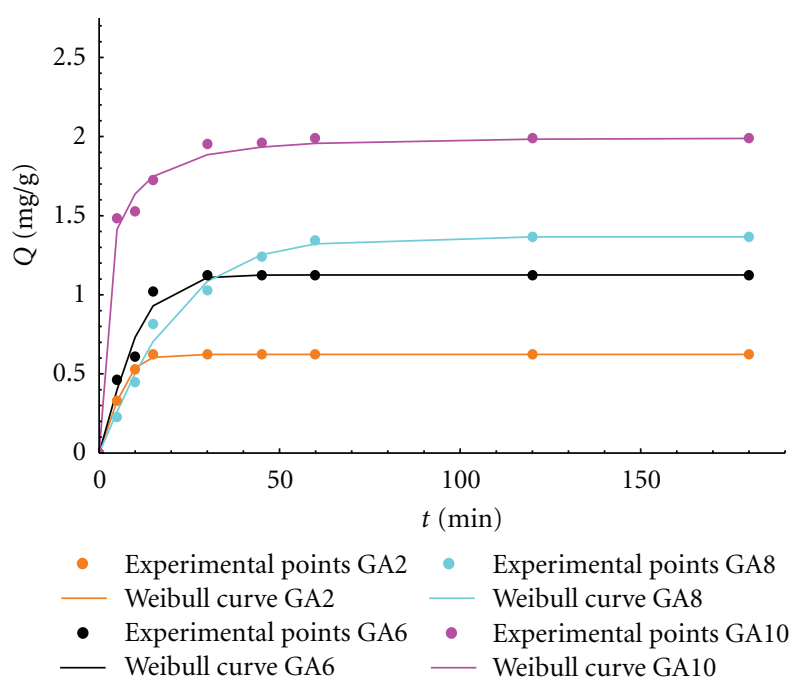

(b)

FIGURE 4: Experimental and Weibull curves for HS samples (a) and GA samples (b).

At the same time, the values for $n$, namely no. 0,5 value, show that in all these cases the diffusion mechanism is a non-Fickian one, while diffusion does not prevail. There are also some other phenomena with relevant contribution: the physical interaction of drug-polymer matrix with release environment, chemical reactions, and drug/polymer degradation.

Consequently, the complexity of the phenomena manifestly generates a complex trajectory for drug particles. It is known that the complex trajectory can be measured through fractal dimension, namely, in this particular case, the fractal dimension of the release curve. This is confirmed by fractal dimension values, according to (36b). The values between 1 and 3 generally correspond to the usually accepted values in the case of fractal processes [37]; higher values denote either fractal dimension should be redefined as a function of structure "classes," or the drug release process 
TABle 1: Values of Peppas and Weibull parameters.

\begin{tabular}{lccccccc}
\hline \multirow{2}{*}{ Sample } & \multicolumn{3}{c}{ Peppas } & \multicolumn{3}{c}{ Weibul } & \multirow{2}{*}{ Fractal dimension } \\
& $k$ & $n$ & Correlation coefficient & $a$ & $b$ & Correlation coefficient & \\
\hline HS4 & 0,6537 & 0,0669 & 0,9816 & 0,4441 & 0,3822 & 0,998 & 5,23 \\
HS5 & 0,5602 & 0,107 & 0,9325 & 0,1715 & 0,6171 & 0,974 & 3,24 \\
HS6 & 0,3623 & 0,1623 & 0,9197 & 0,0525 & 0,7693 & 0,9981 & 2,60 \\
GA2 & 0,6482 & 0,0994 & 0,948 & 0,0782 & 1.4069 & 0,9994 & 1,42 \\
GA6 & 0,4649 & 0,1704 & 0,9246 & 0,0577 & 1.2602 & 0,9902 & 1,59 \\
GA8 & 0,2328 & 0,3079 & 0,9346 & 0,0348 & 1,121 & 0,9953 & 1,78 \\
GA10 & 0,6791 & 0,085 & 0,9896 & 0,5711 & 0,4816 & 0,9966 & 4,15 \\
GC10-90 & & $<\mathbf{0}$ & & & $<\mathbf{0}$ & & \\
GC30-70 & 0,29 & 0,1553 & 0,8564 & 0,2947 & 0,2499 & 0,827 & 8,00 \\
GC50-50 & & $<0$ & & & $<\mathbf{0}$ & & \\
\hline
\end{tabular}

is a complex one, with many freedom degrees in the phase space [38]. Another observation refers to samples with $D_{F}<$ 2 ("subdiffusion") or with $D_{F}>2$, (superdiffusion) (see paragraph 3.). This classification is in compliance to the experimental observation according to which these samples, unlike others, exhibit "faster" diffusion, with higher diffusion rate.

At the same time, the value for fractal dimension of the adrenaline release curve for GC 30-70 sample is the highest, reflecting the high degree of complexity. Consequently, the behavior of such systems should be differently described. Such an approach will be subject of future research. GC samples exhibit strong variations on the first part of the release curve. For two of them, the attempt to match with Peppas and Weibull equations failed, the parameters having negative values that led to negative fractal dimensions (written in bold, in Table 1). These are values that can point to some phase transitions in multiphase complex space [39].

\section{Conclusions}

In this paper, we have replaced the complexity of the physical processes that generates drug release from polymer matrix with fractality. That is why the whole classical "arsenal" of standard physics quantities (differentiable physics) was no longer used. Consequently, fractal approximation of motion was introduced in the study of the complex physic-chemical dynamics of the system. By means of fractional calculus, the fractal "diffusion" equation gives rise to Weibull relation, a statistical distribution function of wide applicability, including drug release studies. Our approach has considered all simultaneously involved phenomena that are equivalent to complexity and fractality. Consequently, a physical basis to this equation and for its parameters has been created. The parameters are functions of fractal dimension of the curves on which drug release mechanism takes place. This dimension measures the complex and nonlinear dynamics of the system that depends on diffusion order.

This type of approach is a viable one, since the analysis of our experimental results shows that experimental curves can match with some very good correlation factors, through Weibull laws, better than in the case of power type laws. At the same time, the fractal dimension of the drug release curve depends on the complexity of the system and can be considered as control parameter for the nonlinear behavior of it.

\section{Acknowledgments}

This paper was supported by PERFORM-ERA ("Postdoctoral Performance for Integration in the European Research Area" (ID-57649)) project, financed by the European Social Fund and the Romanian Government. Thanks to the referent for his pertinent observations.

\section{References}

[1] B. B. Mandelbrot, The Fractal Geometry of Nature, Freeman, San Francisco, Calif, USA, 1983.

[2] D. Stauffer and H. E. Stanle, From Newton to Mandelbrot, Academic Press, New York, NY, USA, 1996.

[3] V. U. Novikov and G. V. Kozlov, "Structure and properties of polymers in terms of the fractal approach," Russian Chemical Reviews, vol. 69, no. 6, pp. 523-549, 2000.

[4] G. V. Kozlov and G. E. Zaikov, Fractals and Local Order in Polymeric Materials, Nova Science Publishers, New York, NY, USA, 2001.

[5] E. Hornbogen, "Fractals in microstructure of metals," International Materials Reviews, vol. 34, no. 6, pp. 277-296, 1989.

[6] M. E. Cates, "Statics and dynamics of polymeric fractals," Physical Review Letters, vol. 53, no. 9, pp. 926-929, 1984.

[7] J. Havlin and D. Ben-Avraham, "Fractal dimensionality of polymer chains," Journal of Physics A, vol. 15, pp. L311-L316, 1982.

[8] M. Muthukumar, "Dynamics of polymeric fractals," The Journal of Chemical Physics, vol. 83, no. 6, pp. 3161-3168, 1985.

[9] M. H. Bessendorf, "Stochastic and fractal analysis of fracture trajectories," International Journal of Engineering Science, vol. 25, no. 6, pp. 667-672, 1987.

[10] Y. Fu and W. J. Kao, "Drug release kinetics and transport mechanisms of non-degradable and degradable polymeric 
delivery systems," Expert Opinion on Drug Delivery, vol. 7, no. 4, pp. 429-444, 2010.

[11] S. Popescu, Actual Issues in the Physics of Self-Structured Systems, Tehnopress, Iasi, Romania, 2003.

[12] L. Nottale, "Fractals and the quantum theory of space-time," International Journal of Modern Physics A, vol. 4, pp. 50475117, 1989.

[13] L. Nottale, Fractal Space-Time and Microphysics: Towards a Theory of Scale Relativity, World Scientific, Singapore, 1993.

[14] K. Kosmidis, P. Argyrakis, and P. Macheras, "Fractal kinetics in drug release from finite fractal matrices," Journal of Chemical Physics, vol. 119, no. 12, pp. 6373-6377, 2003.

[15] P. Costa and J. M. Sousa Lobo, "Modeling and comparison of dissolution profiles," European Journal of Pharmaceutical Sciences, vol. 13, no. 2, pp. 123-133, 2001.

[16] S. Popescu, "Turing structures in dc gas discharges," Europhysics Letters, vol. 73, no. 2, pp. 190-196, 2006.

[17] A. J. Lichtenberg and M. A. Lieberman, Regular and Stochastic Motion, Springer, New York, NY, USA, 1983.

[18] L. Nottale, Scale Relativity and Fractal Space-Time-A New Approach to Unifying Relativity and Quantum Mechanics, Imperial College Press, London, UK, 2011.

[19] R. P. Feynman and A. R. Hibbs, Quantum Mechanics and Path Integrals, MacGraw-Hill, New York, NY, USA, 1965.

[20] D. G. Dimitriu, "Physical processes related to the onset of lowfrequency instabilities in magnetized plasma," Czechoslovak Journal of Physics, vol. 54, supplement, no. C468, p. C474, 2004.

[21] D. G. Dimitriu, V. Ignatescu, C. Ioniţă, E. Lozneanu, M. Sanduloviciu, and R. W. Schrittwieser, "The influence of electron impact ionisations on low frequency instabilities in a magnetised plasma," International Journal of Mass Spectrometry, vol. 223-224, pp. 141-158, 2003.

[22] M. Agop, N. Forna, I. Casian-Botez, and C. Bejenariu, "New theoretical approach of the physical processes in nanostructures," Journal of Computational and Theoretical Nanoscience, vol. 5, no. 4, pp. 483-489, 2008.

[23] I. Casian-Botez, M. Agop, P. Nica, V. Paun, and G. V. Munceleanu, "Conductive and convective types behaviors at nano-time scales," Journal of Computational and Theoretical Nanoscience, vol. 7, no. 11, pp. 2271-2280, 2010.

[24] G. V. Munceleanu, V. P. Paun, I. Casian-Botez, and M. Agop, "The microscopic-macroscopic scale transformation through a chaos scenario in the fractal space-time theory," International Journal of Bifurcation and Chaos, vol. 21, no. 2, pp. 603-618, 2011.

[25] J. F. Gouyet, Physique et Structures Fractals, Masson, Paris, France, 1992.

[26] M. S. El Naschie, O. E. Rössler, and I. Prigogine, Quantum Mechanics, Diffusion and Chaotic Fractals, Elsevier, Oxford, UK, 1995.

[27] P. Weibel, G. Ord, and O. E. Rösler, Space Time Physics and Fractality, Springer, New York, NY, USA, 2005.

[28] K. C. Sung, P. R. Nixon, J. W. Skoug et al., "Effect of formulation variables on drug and polymer release from HPMCbased matrix tablets," International Journal of Pharmaceutics, vol. 142, no. 1, pp. 53-60, 1996.

[29] J. Siepmann, A. Streubel, and N. A. Peppas, "Understanding and predicting drug delivery from hydrophilic matrix tablets using the "sequential layer" model," Pharmaceutical Research, vol. 19, no. 3, pp. 306-314, 2002.

[30] V. Papadopoulou, K. Kosmidis, M. Vlachou, and P. Macheras, "On the use of the Weibull function for the discernment of drug release mechanisms," International Journal of Pharmaceutics, vol. 309, no. 1-2, pp. 44-50, 2006.

[31] L. Jude, Mathematics Physics Equations. Theory and Applications, Matrix Rom Publishing, Romania, 2010.

[32] K. B. Oldham and J. Spanier, The Fractional Calculus: Theory and Applications of Differential and Integration to Arbitrary Order, Dover Publications, New York, NY, USA, 2006.

[33] A. A. Kilbas, H. M. Srivastava, and J. J. Trujilto, Theory and Applications of Fractional Differential Equations, Elsevier, Armsterdam, The Netherlands, 2006.

[34] C. A. Peptu, A. Perichaud, and M. Popa, "Hydrogel microspheres based on environmentally friendly polymers with potential biomedical applications," Environmental Engineering and Management Journal, vol. 10, no. 5, pp. 717-727, 2011.

[35] A. Uliniuc, Chemical modifications of polysaccharides and their hydrogels through "click-chemistry" procedure, Ph.D. thesis, "Ghe. Asachi" Technical University of Iasi, Iasi, Romania, 2011.

[36] C. A. Peptu, G. Buhus, M. Popa, A. Perichaud, and D. Costin, "Double cross-linked chitosan'"Gelatin particulate systems for ophthalmic applications," Journal of Bioactive and Compatible Polymers, vol. 25, no. 1, pp. 98-116, 2010.

[37] P. S. Addison, Fractals and Chaos-An Illustrated Course, Institute of Physics Publishing, Londra, Regno Unito, 1997.

[38] A. J. Lichtenberg, Phase-Space Dynamics of Particle, John Wiley \& Sons, New York, NY, USA, 1969.

[39] M. H. Jensen, G. Paladin, and A. Vulpiani, "Random fractals, phase transitions, and negative dimension spectra," Physical Review E, vol. 50, no. 6, pp. 4352-4356, 1994. 

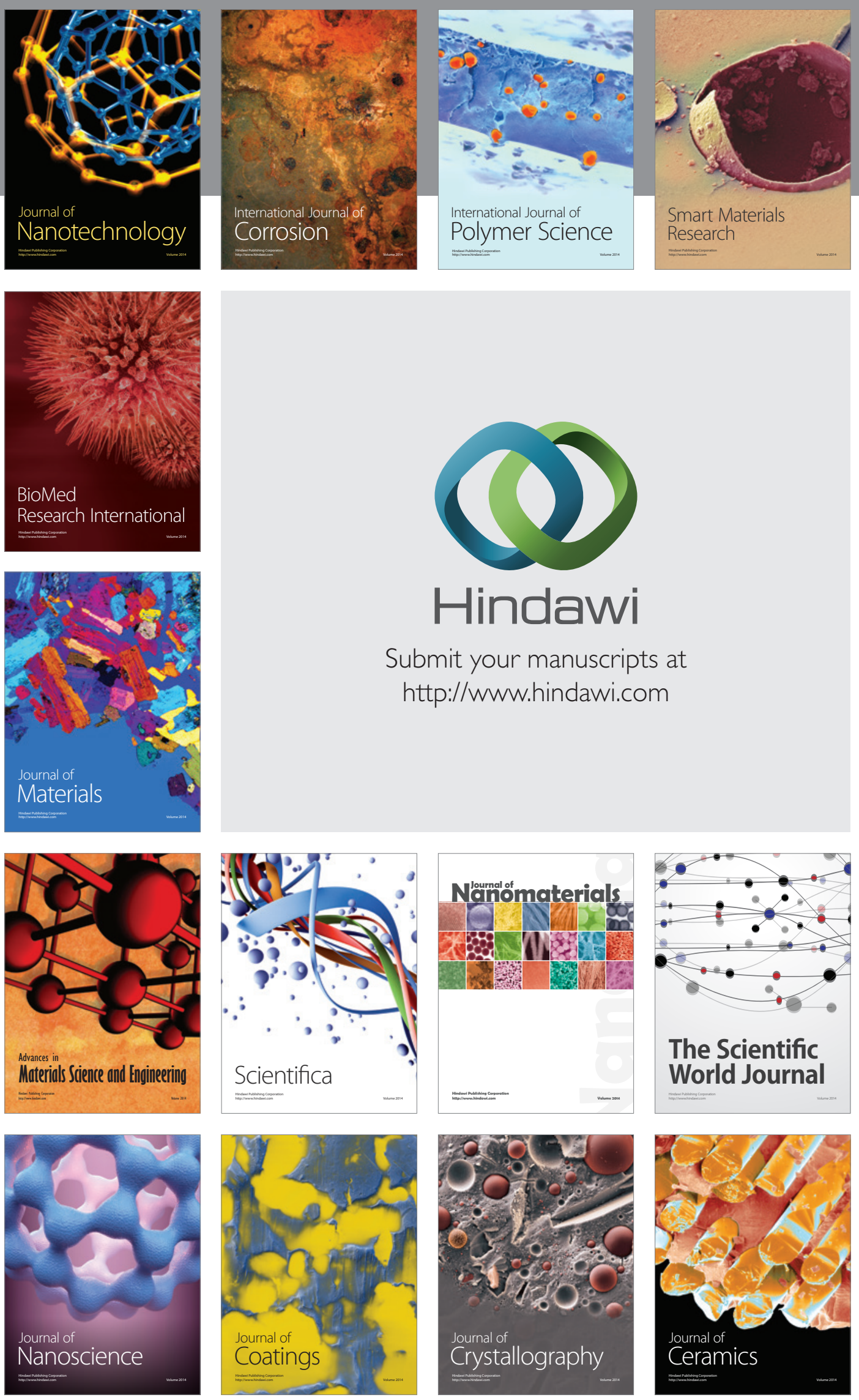

The Scientific World Journal

Submit your manuscripts at

http://www.hindawi.com

\section{World Journal}

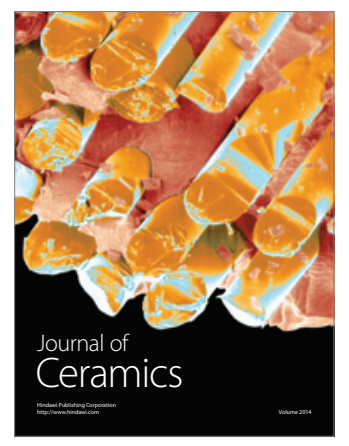

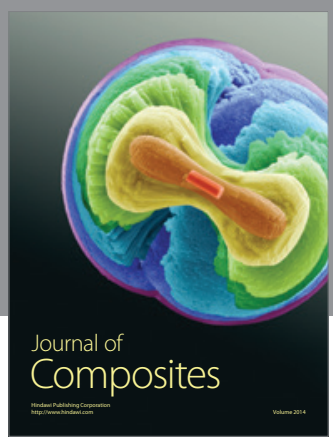
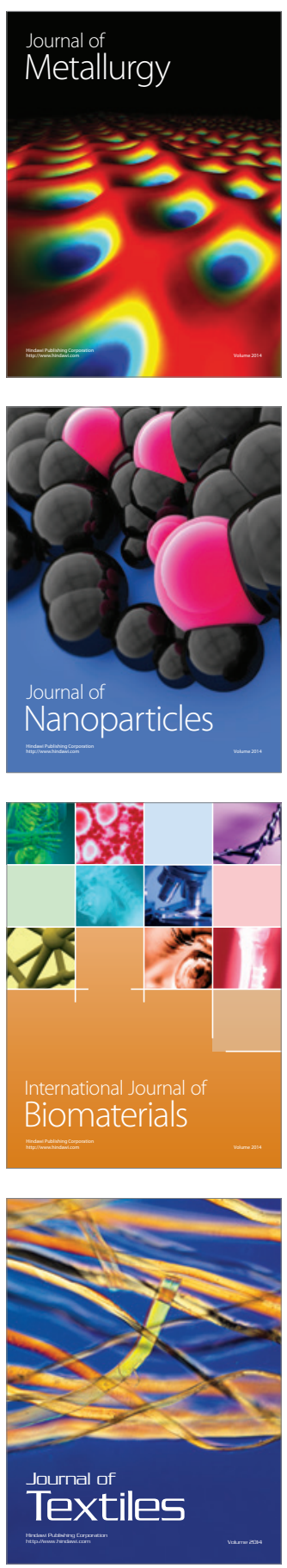